\title{
Genetics and the Etiology of Childhood Cancer
}

\author{
ALFRED G. KNUDSON, JR. (34) \\ Graduate School of Biomedical Sciences, University of Texas Health Science Center, Houston, Texas, USA
}

In developed nations cancer is now the principal cause of death from disease between infancy and adulthood, yet little is known of its etiology. The most uniquely childhood tumors occur so soon after birth in many instances that prenatal initiation becomes suspect. In all parts of the world, each form is uncommon, and, with a few notable exceptions, there is no region with a unique or very unusual incidence of a particular form. In studying the etiology of childhood cancer we begin by suspecting rather universal agents and processes.

\section{WILMS' TUMOR}

Of all the childhood cancers none has a more uniform incidence in the world than Wilms' tumor of the kidney. In fact its incidence of approximately $1 / 15,000$ births is so uniform that its use as an index reference cancer has been proposed (2). It is obviously unpromising to suppose that locally occurring causative factors may be significant contributors to the origin of Wilms' tumor

Still, we are not entirely without etiologic evidence; some high risk groups have been identified. A particularly high risk group is the category of children with sporadic aniridia and various congenital anomalies (25). In at least one case of such a coincidence a chromosomal abnormality (translocation of part of the long arm of a number 11 chromosome to the short arm of a chromosome number 8 , with a deletion in the latter) was found (16). It will be of great interest to discover whether all such cases have some feature of this chromosomal abnormality in common. Another predisposing condition is hemihypertrophy (25), which may also be associated with adrenocortical carcinoma and/or hepatoblastoma. Anomalies of the genitourinary system without hemihypertrophy seem to be associated with Wilms' tumor more often than expected by chance.

Familial cases of Wilms' tumor have also been reported (14) and, in one remarkable family, in association with hemihypertrophy in a parent (22). The familial cases are more often bilateral than usual and are diagnosed at earlier age than is normally the case. The pattern of familial cases is very similar to that which would be expected from retinoblastoma if few affected individuals survived to reproduce; the familial cases are usually twins or other sibs and in only a few instances is more than one generation affected. It has been deduced that there are two large groups of Wilms' tumor patients, one with a hereditary (autosomal dominant) form of the tumor and one with a nonhereditary form (14). An estimate of the fraction of the hereditary form was $38 \%$. The relationship between the two forms was concluded to be similar to that found in retinoblastoma. The prediction was made that as more and more patients survive to adulthood and reproduce there will be more examples of vertical transmission from generation to generation, just as has happened with retinoblastoma.

\section{RETINOBLASTOMA}

Retinoblastoma is distributed on a world-wide basis with an incidence of approximately $1 / 20,000$ births (6). This tumor has long been known to be transmissible in autosomal dominant fashion, but it has also been known that transmission by survivors who had unilateral tumor is much less frequent than by those who had bilateral disease; in fact, in the latter case, transmission fits a dominant gene model. However, the affected offspring of unilateral cases are more often bilaterally affected than not, as with the affected offspring of bilateral cases. The simplest model which explains these observations is one which estimates that approximately $40 \%$ of cases are attributable to a dominant gene which produces a mean number of 3 retinoblastomas/gene carrier, and that it is a matter of chance whether a given individual acquires bilateral or unilateral disease, or, in fact, no disease, as an estimated $5 \%$ of carriers seem to do (9). On the other hand, $60 \%$ of cases occur in children who do not carry such a dominant gene; for these, tumor is a very improbable event and would virtually never occur bilaterally.

These are then two main groups of children who acquire retinoblastoma. One group, with an incidence of about $2 / 100,000$ has a $95 \%$ chance of developing this tumor, and on average develops 3 of them. The other group, which includes essentially all other children, has a probability of about $3 / 100,000$ of developing retinoblastoma. The dominant gene for the tumor increases the number of tumors per individual from 1 primary tumor $/ 30,000$ subjects to 3 primaries in one patient; i.e., it increases the risk of tumor approximately 100,000 times-a potent carcinogen indeed.

By way of comparing retinoblastoma and Wilms' tumor we should note that if there were no successful treatment for retinoblastoma, as has nearly been the case for Wilms' tumor until recent times, the dominant subgroup of retinoblastoma would have been obscured. The clue to heritability would have been the occurrence of the tumor in identical twins, in sibs, and, very occasionally, in more than one generation. These familial cases would have been more frequently bilateral than usual and would have occurred at earlier ages on average. These expectations accord precisely with the observations reported on Wilms' tumor.

Even retinoblastoma has been curable only in modern times. In the past most gene carriers died. Their continued appearance has been due to recurrent mutations in parental germ cells. Even now most cases do not have a positive family history. An important implication for counseling is that all bilateral cases should be considered heritable regardless of family history. For unilateral cases the problem is very difficult; only about $10-15 \%$ are heritable, but that subgroup is not identifiable except in the presence of a positive family history. Means for identifying the hereditary unilateral group are sorely needed.

In rare instances retinoblastoma has been associated with other tumors or with congenital defects. Other tumors are reported at a generally low rate, probably of the order of magnitude of 1 or $2 \%$ (8). However, one tumor, osteogenic sarcoma, dominates this group and seems to occur in about $1 \%$ of persons who carry the dominant retinoblastoma gene. If these individuals receive $x$ irradiation in an attempt to save useful vision, the risk increases. In fact, it has been estimated that with high doses of irradiation the incidence of osteogenic sarcoma of the orbit may rise to $30 \%(29)$; an already greatly increased risk of this tumor is still further increased.

Congenital defects have also been associated with retinoblastoma, but one cluster of defects is particularly outstanding in this respect, viz., those linked with a deletion of the long arm of chromosome 13 , formerly referred to as the D-deletion syndrome. 
The diverse anomalies may reasonably be attributed to deletions of various gene loci, the variability in expression of defects being in large part due to variability in the chormosomal segment which is deleted. Among children with this syndrome there is a high risk of retinoblastoma, and its occurrence is most often bilateral. The simplest hypothesis is that the gene for retinoblastoma is located in the $\mathrm{q} 21$ band of the long arm of chromosome 13, that in some cases of the 13-deletion syndrome it is deleted, and that its deletion results in a risk of retinoblastoma identical with that of the usual dominant mutation (27). The latter is evidently not generally the result of deletion, since cases unassociated with congenital defects generally have normal karyotypes, whether they are unilateral or bilateral (17). Presumably retinoblastoma can ensue whether the gene is changed microscopically (deletion) or not (point mutation or other submicroscopic change). In both cases the gene change is dominantly heritable in the next generation. This same relationship between microscopic and submicroscopic change may be operating in Wilms' tumor, the chromosomal group being comprised of those with aniridia and congenital defects.

\section{NEUROBLASTOMA}

A third important tumor of children which shares many of these features is neuroblastoma. It too is distributed on a world-wide basis, with an incidence of nearly $1 / 10,000$ births. One anomaly in its distribution is a significantly decreased incidence in troprical Africa (6), although its incidence is not decreased in American blacks (24).

In view of the poor survival rate for neuroblastoma it is hardly surprising that familial cases are usually identical twins, sibs and only occasionally members of different generations, just as with Wilms' tumor (13). Here, too, bilaterality, or rather multiplicity (since any portion of the sympathetic nervous system may be affected), and earlier onset relate to familial cases. It has been estimated that more than $20 \%$ of cases are attributable to a dominant gene. In a few cases one family member had ganglioneuroma rather than neuroblastoma, not such a remarkable finding when one considers that these two tumor types may be found in the same tumor mass; ganglioneuroma is evidently a differentiated form of neuroblastoma. This phenomenon of differentiation may account for some instances of vertical transmission of neuroblastoma $(5,7)$. However, for neuroblastoma, as well as for Wilms' tumor and retinoblastoma, this mechanism cannot account for all instances of affected sibs born of unaffected parents. In some cases the parent may have had a nonmanifesting dominant gene, but in others some other mechanism is probably operating. One excellent possibility is gonadal mosaicism, in which a new germinal mutation occurred in a parental gonad early in development, thus giving rise to a large fraction of mutant germ cells and a high probability of more than one affected child. Such an event renders genetic counseling hazardous, especially for retinoblastoma, where the recurrence risk for a second affected child of unaffected parents is of the order of $5-10 \%$.

Neuroblastoma differs from the other two tumors in that no stigmatizing predispositions such as aniridia or 13-deletion syndrome have been identified. Tumors at other sites have been reported, both within and outside fields of radiation (21). Congenital defects have also been reported, but it is not clear that their incidence is excessive, they do not form a unique cluster as with aniridia and Wilms' tumor, nor have they yet been related to a specific chromosomal abnormality as with the 13-deletion syndrome and retinoblastoma. Further investigation of such a possibility should be made.

\section{OTHER SOLID TUMORS OF CHILDHOOD}

For various reasons the other tumors of childhood have not been analyzed in the same way. The analysis of brain tumors, for example, is complicated histologically and some forms have a significant incidence in adulthood. Rhabdomyosarcoma has only been accurately diagnosed on a wide scale in relatively recent times. Hepatic tumors of childhood have a low incidence, as does Ewing's sarcoma. Osteogenic sarcoma and other connective tissue sarcomas have a peak incidence in adolescence and continue to occur in adults. Even so, there are enough published reports to suggest that the same pattern of two groups, one attributable to a dominant gene and one nonhereditary, is operative for these tumors also (15).

The occurrence of brain tumors in sibs is not a common event, but it greatly exceeds the expectation of chance (23). Brain tumors have also been reported in more than one generation and, although no characteristic dominantly inherited forms have emerged, medulloblastoma has an excessive incidence among individuals with the dominantly inherited basal cell nevus syndrome (26).

Rhabdomyosarcoma has also been reported in sibs and in more than one generation, but here a new factor is introduced. Rhabdomyosarcoma has been found in several pedigrees in which breast cancer, and perhaps certain other cancers, have been transmitted through multiple generations (19). These pedigrees deviate from others discussed so far in that the tumor gene is not primarily specific for a childhood tumor.

Deviating even further from this pattern are other somatic tissue sarcomas, including osteogenic sarcoma, fibrosarcoma, and neurofibrosarcoma (4). As noted for retinoblastoma, osteogenic sarcoma may be an associated tumor. It has also been found as a second tumor, either within or outside radiation fields, associated with several other tumors. Fibrosarcoma also has been associated with various tumors, either in the same individual or in different members of the same pedigree. It is well known as a part of the dominantly inherited Gardner's syndrome, a form of polyposis of the colon. Neurofibrosarcoma is best known as a part of dominantly inherited neurofibromatosis, or von Recklinghausen's disease, and a large fraction of these tumors seem to occur in such subjects (4).

Accurate incidence data on these solid tumors are not available, but for brain tumors at least, just as with neuroblastoma, there seems to be a deficiency in parts of Africa (6). In no large population does any of these tumors exceed an incidence of $1 / 10,000$ in childhood, however, and in no large population are they unknown. Reliable incidence data should be obtained before any conclusions can be drawn about the operation of local factors in the etiology and pathogenesis of these tumors.

One generalization that can be made for these tumors, and of most, if not all childhood tumors, is that for each there is a dominantly heritable and a nonhereditary subgroup. The genes responsible for the former increase tumor-specific risks by an order of magnitude of 10,000-100,000 times, often produce more than one tumor, and cause them at earlier than average ages.

\section{LEUKEMIA AND LYMPHOMA}

In childhood this heterogeneous collection of disorders includes two primarily adult forms of leukemia, acute and chronic myeloid, the primarily adult Hodgkin's disease, and a collection of lymphomas which may be observed in child or adult. Two conditions stand out as primarily childhood diseases, acute lymphatic leukemia and Burkitt's lymphoma. The incidence of these two disorders varies drastically in different parts of the world. Acute lymphatic leukemia, for example, is relatively high (about $1 / 3,000$ births) in the United States and much less common in such widely separated places and populations as Japan and Africa. Burkitt's lymphoma, on the other hand, is relatively high in Africa and much less common in the United States and Japan.

Familial leukemia has been observed in both children and adults and has even been reported as occurring over four generations. Twin data show that concordance in identical twins for childhood leukemia approximates $25 \%(20,23)$, which is about the level of concordance estimated for the common solid tumors of childhood. Although a dominant mutant gene could account for these observations, there are no persuasive arguments, partly because data on multiple primaries are not available for leukemia and 
because survival to adulthood from childhood leukemia has not occurred until recent times.

There are also a number of conditions which predispose strongly to leukemia or lymphoma, including the Down syndrome, the syndromes of Fanconi and Bloom, ataxia telangiectasia, and the immune deficiency diseases. Except for the first of these, all are recessive (autosomal or X-linked) disorders. Swift (31), however, has gathered evidence to suggest that heterozygous carriers of the Fanconi mutation are at increased risk for leukemia; in effect, this mutation might represent a mildly penetrant dominant gene for leukemia.

Although no clear picture emerges for heritability of leukemia, there is a distinct possibility that the offspring of the ever increasing list of survivors of childhood leukemia might be at increased risk too.

\section{A MUTATION MODEL OF CHILDHOOD CANCER}

What relationship do the dominantly mutant subgroups of childhood cancers bear to the nonhereditary forms of the same cancers? Are nonhereditary cases formed by an entirely different mechanism, or is there something in common? Another question: what is the germinal mutation in the dominant form doing? All cells in a target tissue bear the mutation, but only a very small number, of the order of magnitude of 1-3 cells, ever become malignant. Obviously, the mutation is not a guarantee that a normal cell will be transformed into a cancer cell. Presumably something else must happen. One attempt to deal with these considerations has resulted in the construction of a model which relates hereditary and nonhereditary cancer $(9-11)$.

The model is very simple. Normal cells are visualized as being capable of undergoing mutation to an intermediate state which is precancerous, but in which differentiation is normal or nearly so. In individuals who carry a dominant cancer mutation all cells in a target tissue are in this state. In individuals who do not carry such a mutation at the stage of fertilization it may arise postzygotically by the process of somatic mutation. Since it is estimated that such mutations occur at frequencies of $1 /$ million or less per cell division, an average normal person may have only a few such cells in the target tissue. However, no mutant cell, whether in a hereditary or in a nonhereditary case, will be transformed into a cancer cell with high probability. In fact, our estimates are that the frequency of this event for childhood tumors is also of the order of magnitude of $1 /$ million or less per cell division-just the frequency of mutation rates in somatic cells. The simplest model is therefore one which hypothesizes that two mutations are necessary to transform a normal cell into a cancer cell. The first of these may occur in either germ cells or in somatic cells, the second in somatic cells. Presumably a cell which acquired both mutations germinally would immediately be a tumor cell and lead to an aborted embryo. As a result there are two classes of individuals with respect to such mutations. One class, relatively rare, acquires one mutation germinally, is relatively infrequent, and develops a specific tumor with high frequency. The other class, comprising most individuals, acquires this first mutation somatically and develops the specific tumor with low frequency. The first class of individuals will be subject to more than one tumor in the target tissue, and possibly to certain other tumors, and will develop tumor earlier on average than will the second class.

Any model applied to childhood cancers must account for the observations that their age-specific incidence show a peak in childhood, and their occurrence in adulthood is rare or uncommon. The most likely reason for these observations is that the tumors arise from embryonal cells which rarely, if ever, persist into adult life. These cells differentiate and in many instances even enter a postmitotic state. In other words, the target cells for transformation into cancer cells are a population of cells disappearing very early in life.

A mathematical model has been constructed by which the total incidence of the two classes of a given tumor, i.e., dominant and nonhereditary, can be estimated (12). The fraction of individuals with the gene for the dominant hereditary form, $f_{g}$, will depend upon the germ cell mutation rate, $\mu_{g}$, and the coefficient of selection, s (a variable which has a value of 0.0 for normal individuals and 1.0 for a totally lethal gene which is never transmitted to offspring), as follows: $f_{g}=2 \mu_{\mathrm{g}} / \mathrm{s}$. The mean number of tumors such persons acquire, $\mu_{\mathrm{g}}$, will be distributed in a chance

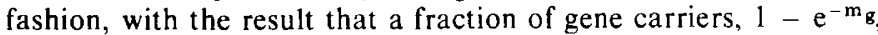
develop at lease one tumor. From this it follows that the incidence of the hereditary form, $\mathrm{i}_{\mathrm{g}}$, is: $\mathrm{i}_{\mathrm{g}}=\mathrm{f}_{\mathrm{g}}\left(1-\mathrm{e}^{-\mathrm{m}_{\mathrm{g}}}\right)=2 \mu_{\mathrm{g}} / \mathrm{s}\left(1-\mathrm{e}^{-\mathrm{m}_{\mathrm{g}}}\right)$ $=\mathrm{a} \cdot \mu_{\mathrm{g}}$.

In nonhereditary cases we may state that the population at risk is $1-f_{g}$, which is essentially unity for childhood cancer. If the mean number of tumors in nonhereditary cases is $m_{n}$, then $i_{n}$, the incidence of nonhereditary cases, is related to $m_{n}$ as follows: $i_{n}=1$ $-\mathrm{e}^{-\mathrm{m}_{\mathrm{n}}}$, which reduces, since $m_{n}<<1$, to: $i_{n}=m_{n}$. The mean number of tumors in the nonhereditary form is determined by the mutation rate, $\mu_{\mathrm{s}}$, for the first mutation; $\mathrm{m}_{\mathrm{n}}=\mathrm{b} \cdot \mu_{\mathrm{s}}$. From these expressions it follows that the incidence of a childhood cancer takes the form: $\mathrm{i}=\mathrm{i}_{\mathrm{g}}+\mathrm{i}_{\mathrm{n}}=\mathrm{a} \cdot \mu_{\mathrm{g}}+\mathrm{b} \cdot \mu_{\mathrm{g}}$. Therefore, the incidence of a childhood cancer is dependent upon germinal and somatic mutation rates.

\section{CONSEQUENCES OF MUTATION MODEL}

This mutation model, and one may say, generally, any mutation model, relates the incidence of a particular childhood cancer to mutation rates in germinal and somatic cells. This relationship creates an expectation for a minimal incidence of cancer, which in the absence of demographic variation in mutation rates should be constant from one part of the world to another. Since the mutation rates in the above expressions are almost certainly smaller than $10^{-5}$, and since the constants, a and $b$, are not greater than 10 , the incidence of any childhood tumor should be less than $1 / 10,000$. In fact, this is the case for childhood cancers except for leukemia and lymphoma. Whether these latter diseases follow some other model or whether mutation rates can be unusually high in their precursor cells is not known.

It is also apparent that any decrease in the coefficient of selection for a hereditary tumor can result in an increase in the incidence of the hereditary form. This has probably been the case for retinoblastoma in this century and may be a result of the presently successful treatment of Wilms' tumor.

The mutation rates in these expressions are "spontaneous" or "background" mutation rates for germinal and somatic cells. Of course they can be increased by environmental mutagens for any gene. For this reason we should not be surprised to find that $\mathrm{x}$-irradiation may lead to an increase in a particular form of cancer. For childhood cancers this seems to be especially important when the irradiation occurs prenatally (30). In connection with retinoblastoma it was also noted that "second cancers" are more common in irradiated areas, further supporting the notion that the second event in transformation is also a mutation. Evidence that irradiation, whether ionizing or ultraviolet, can cause cancer via a mechanism of mutation has been provided in the case of ultraviolet light by explication of the defect in xeroderma pigmentosum. Here a Mendelian recessive disorder predisposes to cancer by virtue of a defect in the repair of ultraviolet-induced damage to DNA, i.e., to an increased mutation rate for a given dose of ultraviolet light.

Chemical carcinogens, in most if not all cases, also seem to exert their effects by increasing mutation rates (1). Although there is no reason to suspect that such carcinogens contribute any significant fraction of childhood cancers, they seem to be important for some adult cancers, especially bronchogenic carcinoma.

The even distribution of some childhood tumors over the world suggests that either environmental mutagens play a very minor role in their origin or they play an equally prominent role everywhere. Also playing a minor role is xeroderma pigmentosum, which increases mutation rates by genetic means. Other conditions which may be in this category are the recessively inherited syndromes of Bloom and Fanconi. There is one claim that the 
latter involves a defect in DNA repair of induced damage (28). But these syndromes are rare and can hardly account for a significant fraction of childhood cancer, although, as noted previously, heterozygotes for the Fanconi syndrome may be susceptible to cancer and contribute significantly to its incidence.

Also noted earlier was the susceptibility to leukemia and lymphoma imparted by the immune deficiency diseases and by certain chromosomal disorders. These do not contribute any measurable increase in the other childhood cancers.

For most solid tumors germinal and somatic mutations could account for the observed incidences. Exceptions may be the decreased incidence of brain tumors and neuroblastoma in parts of Africa and of Ewing's sarcoma in blacks, whether American or African. With respect to the latter observation, no explanation is forthcoming. For the former there is the interesting possibility that some agent indigenous to the area interferes with the growth and survival of these tumors when they do occur.

\section{CAN VIRUSES CAUSE CHILDHOOD CANCER?}

The incidences of leukemia and lymphoma are not readily explained. Although the low incidence of acute lymphatic leukemia in children in Japan might be explained by mutation, what might explain its much higher incidence in the United States and Europe? A popular hypothesis is that some or all of childhood leukemia is caused by a leukemia virus, and, in particular, a virus introduced postzygotically (3). If this were correct, then significant preventive measures might be developed. If this notion is correct, however, the virus must only very occasionally cause leukemia, because authentic "outbreaks" of leukemia are extremely rare if, indeed, they occur at all.

Another possibility is that tumor viruses are acquired prezygotically by virtue of their integration into the host's genome. This model for carcinogenesis cannot be examined in detail here, but it should be noted that very few pedigrees show vertical transmission of human leukemia. If such a viral genome causes human leukemia it must only occasionally do so. One possibility is that an integrated viral genome must undergo modification before it produces cancer. Under these circumstances the difference between a mutational and a viral theory of cancer becomes nebulous.

\section{SUMMARY}

A consideration of the world-wide incidences of childhood cancer and of hereditary subgroups leads to the conclusion that two successive mutations can initiate cancer cells and that such cells usually proceed to develop into detectable cancers in a period of time which is short compared with the time required for most adult cancers. Environmental carcinogens could hypothetically increase the rates at which these mutations occur, but they probably, in fact, contribute little to the incidences. Certain exceptions, notably leukemia and lymphoma, are noteworthy, and a viral origin for them has been widely hypothesized.

If most solid tumors of childhood are indeed correctly attributable to mutations in germ and/or somatic cells, then the prospect for the prevention of childhood cancer becomes very dim. In fact, the incidence of the germinal forms may increase as treatmen improves (18). In theory, one might be able to identify individuals harboring cancer genes germinally and even to identify them prenatally. But even if the burden of cancer attributable to the hereditary subgroups were eliminated, there would still remain the larger nonhereditary group resulting from somatic mutations. If this hypothesis is correct, then childhood cancer cannot be prevented. With this conclusion goes the admonition, however, that environmental mutagens might significantly increase the burden of childhood cancer. One such mutagen, therapeutic radiation, is known to increase the prospect that second tumors will occur in patients who carry a germinal cancer mutation. The major effort to reduce the incidence of childhood cancer by prevention should be spent in examining the possibility that leukemia and lymphoma are viral in origin.

If the arguments presented are correct, then the main effort against childhood cancer must be that of early diagnosis and treatment. I realize that many have already argued for that strategy in the approach to cancer generally, but I now believe that it is particularly relevant to any program against cancer in children.

\section{REFERENCES AND NOTES}

1. Ames, B. N. Durston, W. E., Yamasaki, E., and Lee, F. D. Carcinogens are mutagens: A simple test system combining liver homogenates for activation and bacteria for detection. Proc. Natl. Acad. Sci. U. S. A., 70: 2281 (1973).

2. Anonymous: Nephroblastoma: An index reference cancer. Lancet, ii: 651 (1973).

3. Baxt, W. Yates, J. W., Wallace, H. J., Holland, J. F., and Spiegelman, S.: Leukemia-specific DNA sequences in leukocytes of the leukemic member of identical twins. Proc. Natl. Acad. Sci. U. S. A., 70: 2629 (1973).

4. Chabalko. J. J., Creagen, E. T., and Fraumeni, J. F.: Epidemiology of selected sarcomas in children. J. Natl. Cancer Inst., 53: 675 (1974).

5. Chatten, J and Voorhess, M. L.: Familial neuroblastoma: report of a kindred with multiple disorders, including neuroblastoma in four siblings. $N$. Engl. $J$. Med., 277: 1230 (1967).

6. Davies, J. N. P.: Childhood tumours. In: A. C. Templeton: Tumours in a Tropical County: A Survey of Uganda 1964-1968, Chap. 19, pp. 306-343 (SpringerVerlag, New York 1973).

7. Gerson, J. M., Chatten, J., and Eisman, S.: Familial neuroblastoma-A follow-up. N. Engl. J. Med., 290: 1487 (1974).

8. Kitchin, F. D., and Ellsworth, R. M.: Pleiotropic effects of the gene for retinoblastoma. J. Med. Genet., 11: 244 (1974).

9. Knudson, A. G.: Mutation and cancer: statistical study of retinoblastoma. Proc. Natl. Acad. Sci. U. S. A., 68: 820 (1971).

10. Knudson, A. G.: Mutation and human cancer. Advan. Cancer Res., 17: 317 (1973).

11. Knudson, A. G.: Heredity and human cancer. Amer. J. Pathol. 77: 77 (1974)

12. Knudson, A. G., Hethcote, H. W., and Brown, B. W.: Mutation and childhood cancer: a probabilistic model for the incidence of retinoblastoma. Proc. Natl. Acad. Sci. U. S. A., 72: 5116 (1975).

13. Knudson, A. G., and Strong, L. C.: Mutation and cancer: Neuroblastoma and pheochromocytoma. Amer. J. Hum. Genet., 24: 514 (1972).

14. Knudson, A. G., and Strong. L. C.: Mutation and cancer: A model for Wilms tumor of the kidney. J. Natl. Cancer Inst., 48: 313 (1972)

15. Knudson, A. G., Strong, L. C., and Anderson, D. E.: Heredity and cancer in man Progr. Med. Genet., 9: 113 (1973).

16. Ladda, R., Atkins, L., Littlefield. J., Neurath, P., and Marimuthu, K. M Computer-assisted analysis of chromosomal abnormalities: Detection of a deletion in aniridia/Wilms' tumor syndrome. Science, 185: 784 (1974).

17. Ladda, R., Atkins, L., Littlefield, J., and Pruitt, R.: Retinoblastoma: Chromosome banding in patients with heritable tumour. Lancet, ii: 506 (1973).

18. Lewis, E. B.: Possible genetic consequences of irradiation of tumors in childhood. Radiology, 114: 147 (1975)

19. Li, F. P., and Fraumeni, J. F.: Soft-tissue sarcomas, breast cancer, and other neoplasms. Ann. Int. Med., 71: 747 (1969)

20. MacMahon, B., and Levy, M. A.: Prenatal origin of childhood leukemia: Evidence from twins. N. Engl. J. Med., 270: 1082 (1964)

21. Meadows, A. T., D'Angio, G. J., Evans, A. E., Harris, C. C., Miller, R. W., and Mike, V.: Oncogencsis and other late effects of cancer treatment in children. Radiology, 114: 175 (1975)

22. Meadows, A. T.. Lichtenfeld, J. L., and Koop, C. E.: Wilms' tumor in three children of a woman with congenital hemihypertrophy. N. Engl. J. Med., 291: 23 (1974)

23. Miller, R. W.: Deaths from childhood cancer in sibs. N. Engl. J. Med., 279: 122 (1968).

24. Miller, R. W., and Dalager, N. A.: U. S. childhood cancer deaths by cell type, 1960 68. J. Pediat., 85: 664 (1974).

25. Miller, R. W., Fraumeni, J. F., and Manning, M. D.: Association of Wilms tumor with aniridia, hemihypertrophy and other congenital malformations. $\mathrm{N}$. Engl. J. Med., 270: 922 (1964)

26. Neblett, C. R., Waltz, T. A., and Anderson, D. E.: Neurological involvement in the nevoid basal cell carcinoma syndrome. J. Neurosurg., 35: 577 (1971),

27. Orye, E., Delbeke, M. J., and Vandenabeele, B.: Retinoblastoma and long arm deletion of chromosome 13. Attempts to define the deleted segment. Clin. Genet. 5: 457 (1974).

28. Poon, P. K. O'Brien, R. L., and Parker, J. W.: Defective DNA repair in Fanconi's anaemia. Nature, 250: 223 (1974)

29. Sagerman, R. H., Cassady, J. R., Tretter, P., and Ellsworth, R. M.: Radiation induced neoplasia following external beam therapy for children with retinoblastoma. Amer. J. Roentgenol., I05: 529 (1969).

30. Stewart, A., Webb. J., and Hewitt, D.: A survey of childhood malignancies. Brit. Med. J., $l: 1495$ (1958)

31. Swift, M.: Fanconi's anaemia in the genetics of neoplasia. Nature, 230: 370 (1971). 
32. This paper was presented at a Symposium on Childhood Cancer at the Annual Meeting of the American Pediat ric Society, Denver, Colorado, April 17, 1975.

33. This research was supported in part by Medical Genetics Center Grant no. Center, Houston, Tex. 77030 (USA).
34. Requests for reprints should be addressed to: A. G. Knudson, Jr., M.D., Graduate School of Biomedical Sciences, University of Texas, Health Sciences Center, Houston, Texas 77030 (USA).

35. Accepted for publication July $25,1975$.

\title{
The Role of Cellular Immunity in Neoplasia
}

\author{
PHILIP R. GLADE(94) AND NIEVES M. ZALDIVAR \\ Division of Pediatric Infectious Diseases and Immunology, University of Miami School of Medicine, \\ Miami, Florida, USA
}

LLOYD MAYER AND LINDA T. CAHILL

Mount Sinai School of Medicine, New York, New York, USA

In the past 15 years we have witnessed a rapid evolution in our understanding of the lymphocyte and its role in health and disease. Whereas peripheral lymphocytes had been viewed traditionally as short lived cells with limited biologic activity, it has become abundantly clear that the circulating lymphocyte pool is composed of a spectrum of subpopulations of cells of varying origins, lifespans, fine structural features, and capacities to mediate immunologic responses $(24,25)$. The possibility that immune mechanisms may prevent the development of potentially malignant cells, first formulated by Ehrlich at the turn of the century (18), is one of the most significant outgrowths of this renewed interest in the lymphocyte. The concept of immunologic surveillance, as proposed by Thomas (80) and Burnet (12), suggests that small numbers of tumor cells with altered surface antigens frequently develop in long lived vertebrates, that these cells are recognized as foreign by the cellular immune system of the host, and that they subsequently are eliminated by immunologic mechanisms. Strong supporting evidence for the assumption that neoplastic transformation is accompanied by altered biochemical processes and by the appearance of new surface antigens has been amply provided by our panelists (92). In this segment we will discuss evidence that the tumor-bearing host can recognize and respond to these neoplastic changes. We will consider some aspects of immunosurveillance, the appropriateness of the immune responses detected in the cancer patient, and the potential augmentation of these responses by immunotherapy. In keeping with our charge, these comments will for the most part be limited to a consideration of $\mathrm{T}$ lymphocyte-mediated immune responses.

Clinical evidence that immunologic mechanisms may function in man in the control of cancer comes from multiple sources, most of which indicate a higher incidence of cancer in individuals with depressed or ineffective immunosurveillance. Prior to chemotherapy, patients with malignant disorders frequently manifest a variety of immunologic deficiencies (72). A high incidence of cancer is also noted at the extremes of age when it can be shown that immune systems are less than optimal (12). It has been suggested that in early life the developing and unprimed immune system receives a large battery of antigenic stimulation by "strong" as well as "weak" antigens in the environment. During this time of antigenic bombardment a weakly antigenic malignant cell could escape recognition and establish itself as a tumor. Alternatively, the relatively naïve responses of the infant may be more easily overwhelmed by the massive antigenic assault produced by rapidly growing tumor cells. Immunologic responsiveness tends to deteriorate with old age, perhaps (as suggested by Goldstein (23)) weakened by thymic atrophy and decreasing thymosin levels. Of considerable importance is the markedly increased incidence of malignancy in patients with primary immunodeficiency disorders. Neoplasms in these groups occur roughly 10,000 times more frequently than they do in the general age-matched population (21). Cancers affect up to $10 \%$ of patients with certain immunodeficiencies, particularly those involving thymus-dependent $T$ lymphocytes. The development of malignancy is significantly increased in individuals with secondary immunodeficiencies as well.

Patients who have undergone prolonged immunosuppressive therapy for the treatment of disease (including cancer) or to prevent rejection of organ transplants have an incidence of cancer 100 times greater than that of the general population (61). That effective immunosurveillance may be the critical factor in the limitation and final elimination of human cancers is further suggested by the multiple clinical observations of spontaneous regression of established tumors with evidence that tumor immunity has subsequently developed (13). The local accumulation of lymphocytes in certain tumors and, especially, the positive correlation between the intensity of the cellular response and the length of survival provide indirect evidence of in vivo host immune responses to tumors in man (6).

In addition to these clinical observations suggesting lymphocyte participation in the immunosurveillance of human neoplasms is direct evidence of in vivo and in vitro cell-mediated immune responses to a variety of tumor-associated antigens $(31,69)$. Studies have indicated the variable presence of delayed-type hypersensitivity skin reactions to autologous extracts of a variety of human tumors including carcinomas (74), Burkitt's lymphoma (19) and leukemias $(47,60)$. Homologous tumor extracts, however. usually have failed to produce similar skin test reactivity, particularly in patients with Burkitt's lymphoma and leukemia. Reactive 